mean a major loss of working years and therefore an advancement of age of disability in the coming years.

\section{5-YEAR TRAJECTORIES OF EMPLOYMENT IN PEOPLE WITH SPINAL CORD INJURY}

${ }^{1}$ A Ferdiana, ${ }^{2}$ Post, ${ }^{3}$ Hoekstra, ${ }^{1}$ van der Klink, 'Bultmann. 'Groningen, The Netherlands; ${ }^{2}$ De Hoogstraat, Utrecht, The Netherlands; ${ }^{3}$ Department of Epidemiology and Biostatistics Vrije University, Amsterdam, The Netherlands

\subsection{6/oemed-2013-101717.325}

Objectives To identify the employment rate over time, to examine employment trajectories and to determine the predictors of distinct employment trajectories in a cohort of people with spinal cord injury (SCI) in The Netherlands.

Methods The study population consisted of 179 participants from eight rehabilitation centres who had acute SCI, were 1865 years old, wheelchair dependent, and able to understand Dutch. At the start of rehabilitation, socio-demographics (age, gender, education), pre-injury occupation (level and physical intensity), injury-related factors (age at onset, neurological level and motor completeness), and functional status were measured. Employment status was defined as " $\geq 1$ hour of paid work/week". Follow-up measurements were conducted 1,2 and 5 years after discharge. Employment trajectories were modelled with longitudinal latent class analysis. Determinants of distinct trajectories were identified using multivariable logistic regressions.

Results The employment rate was $79.5 \%$ before injury, $28.2 \%$ 1 year, $35.1 \% 2$ year and $44.8 \% 5$ year after discharge. Three distinct employment trajectories were identified: 1) neverworked $(\mathrm{N}=35 ; 20 \%)$, those who did not work pre- and postinjury, 2) slow return to work (RTW) $(\mathrm{N}=56 ; 30.9 \%)$, those who worked pre-injury and had a slow RTW, and 3) fast RTW $(\mathrm{N}=88: 49.1 \%)$, those who worked pre-injury and had a fast and sustained RTW. Compared to participants with slow RTW, those with fast RTW had a higher functional status $(\mathrm{OR}=1.07$, $95 \% \mathrm{CI}=1.02-1.12$ ) and a pre-injury occupation with low physical intensity $(\mathrm{OR}=9.83,95 \% \mathrm{CI}=2.27-42.57)$. The model explained $43 \%$ of the variance in RTW trajectory.

Conclusion The employment rate increased over time after discharge. Three distinct employment trajectories were identified. Predictors of fast RTW were higher functional status and preinjury occupation with low physical intensity. Future research should focus on identifying environmental factors that may contribute to a favourable RTW trajectory.

\section{SOCIAL POSITION AND INTELLIGENCE: WHICH IS THE MORE IMPORTANT DETERMINANT OF SICKNESS ABSENCE IN A LIFE-COURSE PERSPECTIVE?}

'S Mehlum, ${ }^{2}$ Susser, ${ }^{2}$ Link, ${ }^{1}$ Corbett, ${ }^{1}$ Kristensen. ${ }^{1}$ National Institute of Occupational Health, Oslo, Norway; ${ }^{2}$ Department of Epidemiology, Mailman School of Public Health, Columbia University, New York City, United States of America

\subsection{6/oemed-2013-101717.326}

Objectives Socio-economic position (SEP) and intelligence (IQ) are both predictors of later health, however, the relationships between them have been much debated. Our objective was to examine the separate effects of IQ and SEP on health, as measured by sickness absence (SA).

Methods All live-born males in Norway 1967-1971 ( $\mathrm{N}=170$ 678) were followed up in several national registers. Our study included subjects who were healthy at age 18-19 years, and at risk of SA at start of follow-up $(\mathrm{N}=99738)$. Health outcome was the 4-year risk (2000-2003) of at least one SA episode. IQ test scores were recorded at military conscription (age 18-19 years). Education level at age 28 and income in 2000 served as indicators of adult SEP, whereas parental education level and income during childhood were indicators of childhood SEP. Risk ratios (RRs) adjusted for birth year were estimated using generalised linear models. Direct and total effects were calculated according to our model.

Results A total of 23506 subjects (24\%) had SA episodes during follow-up. There were strong gradients according to participants' $I Q$, educational attainment, and income. RRs between the lowest and highest of 5 categories were 5.99, 9.05, and 3.36, respectively. The effects were somewhat weakened when adjusted for childhood SEP, and were further reduced when IQ, education and income were all included in the analysis, to calculate the direct effects (RRs 1.84, 4.79, and 1.65, respectively). According to our model, the total effect of IQ (adjusted for childhood SEP), was RR 4.15 .

Conclusions These results suggest that education had a stronger direct effect on health than IQ and income. However, the total effect of IQ was of similar strength and was in part mediated through adult SEP.

\section{A COMPARTMENTAL HIDDEN MARKOV MODEL FOR THE LONGITUDINAL ANALYSIS OF THE RISK OF SMOKING-INDUCED LUNG CANCER}

${ }^{1}$ Chadeau-Hyam, ${ }^{2}$ Vermeulen, ${ }^{3}$ Vineis. ${ }^{1}$ Imperial College London, London, United Kingdom; ${ }^{2}$ Institute for Risk Assessment Sciences, Utrecht, The Netherlands; ${ }^{3}$ Imperial College, London, United Kingdom

\subsection{6/oemed-2013-101717.327}

To account for the dynamic aspects of carcinogenesis, we propose a compartmental hidden Markov model in which individuals are either healthy, asymptomatically affected, diagnosed, or deceased. Our model is illustrated using the example of smoking-induced lung cancer.

The model was fitted on a case control study nested in the European Prospective Investigation into Cancer and Nutrition study including 757 incident cases and 1524 matched controls. Model estimation was done through a Markov Chain Monte Carlo algorithm, and predictive abilities of the model were assessed through a simulation study based on the posterior estimates of the model parameters. We considered a logistic function for the risk of entering carcinogenesis. Sensitivity analyses to assess the role of each of model parameters was performed by comparing sub-models on the basis of their (simulated) predictive performances.

We found that once adjusted on its impact on exposure duration, age does not independently drive the risk of lung carcinogenesis, while age at starting smoking in ever smokers, and time since cessation in former smokers were found influential. We estimated the time between onset of malignancy and clinical diagnosis to range from 2 to 4 years. Our approach yielded good performances in reconstructing individual trajectories in both cases (sensitivity $>90 \%$ ) and controls (sensitivity >80\%). Results also showed that our data did no support an age-dependent time to diagnosis.

The flexible and general formulation of our compartmental model enables the future incorporation of disease states 
measured by intermediate markers into the modelling of the natural history of cancer. Together with its ability to elucidate temporal effects of exposure on disease risk, this suggests a large range of applications in chronic disease epidemiology.

\section{Session: 20. Risk modelling}

\section{MODELLING COMPLEX MIXTURES IN EPIDEMIOLOGIC ANALYSIS: ADDITIVE VERSUS RELATIVE MEASURES FOR DIFFERENTIAL EFFECTIVENESS}

${ }^{1} \mathrm{G}$ B H Hamra, ${ }^{2} \mathrm{R}$ F M Maclehose, ${ }^{3} \mathrm{D} B \mathrm{R}$ Richardson, ${ }^{4} \mathrm{~S} B$ Bertke, ${ }^{4} \mathrm{R} D \mathrm{D}$ Daniels. ${ }^{1}$ University of North Carolina at Chapel Hill School of Public Health, Chapel Hill, United States of America; ${ }^{2}$ University of Minnesota-Epidemiology, Minneapolis, United States of America; ${ }^{3}$ University of North Carolina, Chapel Hill, United States of America; ${ }^{4}$ NIOSH, Cinncinati, United States of America

\subsection{6/oemed-2013-101717.328}

Mixed exposures are often combined into single exposure measures using weighting factors. An example of this arises in radiation epidemiology where doses of distinct forms of ionising radiation (such as alpha, beta, and gamma radiation) are combined based on knowledge of their biological effectiveness relative a reference form of radiation (most often gamma). Similar pooling of mixed exposures may occur with multiple congeners or air pollutants to develop more parsimonious models. The weights used for combining exposures are determined from experimental animal and cellular research, but not observational research. In this work, we show that these weights, which are the ratio of two normally distributed variables, cannot be reliably estimated from observational research. We propose an alterative approach for estimating differences in effectiveness of distinct exposures based on their excess effectiveness compared to a reference exposure. This alternative provides reliable estimates of differences in effectiveness of distinct exposures.

\section{IMPROVING COUNTER-MATCHING DESIGN IN NESTED CASE-CONTROL STUDY TO ANALYSE THE EFFECT OF A CONTINUOUS OCCUPATIONAL EXPOSURE}

D D Drubay, Ancelet, Laurier, Rage. IRSN, Fontenay-aux-Roses, France

\subsection{6/oemed-2013-101717.329}

Introduction Nested case-control studies are classically used in epidemiology to reduce time and cost for data collection while minimising bias induced by sample size reduction. However, if the continuous exposure of interest have a highly skewed probability distribution, rare exposure values are less likely to be selected. Therefore, the sampling process can miss important information in the long tail of the exposure distribution. Counter-matching design attempt to maximise the information on known exposure of interest during sampling process by the selection of controls in several categories covering all the range of the exposure. This study attempt to assess the influence of the choice of the categorisation method on the risk estimation. Method The categorisation of continuous exposure is provided by several classification or clustering Methods: a priori thresholds, quantiles and k-means. To investigate the robustness of these methods, a simulation study is realised with several shapes of the probability distribution of the exposure, addition of covariate and missing collected data. Theoretical results will be illustrated with the example of a case-control study of cardiovascular diseases mortality (440 cases, 5 controls per cases (1:5)) nested within the French cohort of uranium miners (including 5086 men followed-up between 1945 and 2007) where cases and controls are matched on age and birth cohort and counter-matched on cumulative radon exposure.

Results 5000 samples 1:5 have been generated from the French uranium miners cohort for each methods applied on the cumulated radiation exposure. Relatively to the risk estimated from the cohort, the bias was systematically lower by using the kmeans method.

Perspectives Results of the simulation study and application to the case-control study nested within the French uranium miners cohort could confirm this trend and contribute to the improvement of the efficiency of nested case-control study for the assessment of risk of exposure.

\section{A DYNAMIC POPULATION-BASED MODEL FOR THE DEVELOPMENT OF WORK-RELATED RESPIRATORY HEALTH EFFECTS AMONG MOTOR VEHICLE REPAIR WORKERS EXPOSED TO ISOCYANATES}

${ }^{1} \mathrm{R}$ H Klein Entink, ${ }^{1}$ Meijster, ${ }^{1}$ Pronk, ${ }^{2}$ Tan, ${ }^{2}$ Warren, ${ }^{3}$ Heederik. ${ }^{1} T N O$, Zeist, Nederland; ${ }^{2}$ Health and Safety Laboratory, Buxton, United Kingdom; ${ }^{3}$ Utrecht University, Institute for Risk Assessment Sciences, Utrecht, Nederland

\subsection{6/oemed-2013-101717.330}

Objectives Spray painters, who are exposed to isocyanate mixtures, are among the occupational groups with the highest incidence of occupational asthma in industrialised countries. In the Netherlands the motor vehicle repair (MVR) industry comprises one of the largest occupational populations potentially exposed to isocyanates. We present a mathematical model which simulates a population of spray painters exposed to isocyanates longitudinally through time and tracks the development of symptoms in each worker.

Methods First, based on the literature a conceptual disease model was defined which differentiates between different severities of symptoms: healthy, upper airway symptoms only, lower airway symptoms only, both upper and lower airway symptoms, and work-disabling symptoms, where all states are stratified on sensitisation. A Weibull survival analysis of data from an epidemiological study of 424 workers in the Dutch MVR industry confirmed the relationships of respiratory symptoms with isocyanate exposure and atopy. Furthermore, the data provided us with the necessary task-based exposure distributions, patterns of work and use of respiratory equipment that served as inputs for the simulation model.

Results Individual workers had the highest chance of developing respiratory symptoms within the first five years of working in the MVR sector, after which their risks decrease. Furthermore, the risk of developing lower airway symptoms after 5 years doubled when a worker was atopic. The following example illustrates the application of the modelling approach: The prevalence of lower airway symptoms found in the epidemiological study was $5 \%$. On average, $93 \%$ of spray painters used respiratory protection equipment. The dynamic population model predicted that when frequency of use increases to $98 \%$, the prevalence of lower airway symptoms would decrease to $4 \%$. 